\title{
High Risk Factors of Cardiovascular Diseases in Type 2 Diabetes
}

\section{Srilatha B}

Presidency College, Bangalore University, India

\begin{abstract}
Diabetes mellitus is a group of metabolic diseases characterized by hyperglycemia and hypoglycemia that result from defects in insulin secretion. The prevalence of type 2 diabetes mellitus is rapidly increasing all over the world. Cardiovascular disease (coronary heart disease, stroke, Myocardial infarction) is the most important cause of mortality and morbidity among patients with type 2 diabetes. The accelerated Atherosclerosis and cardiovascular disease in diabetes is likely to be multifactorial and therefore several therapeutic approaches can be considered. The number of people with type 2 diabetes is increasing due to population growth, aging, obesity, hypertension, unhealthy diet, smoking and alcohol consumption and increasing urbanization and physical inactivity.
\end{abstract}

Keywords: Diabetes Mellitus; Hyperglycemia; Hypoglycemia; Cardiovascular Diseases; Obesity; Hypertension; Alcohol Consumption.

Abbreviations: HDL: High Density Lipoproteins; LDL: Low Density Lipoproteins; BMI: Body Mass Index;

\section{Introduction}

Diabetes mellitus, a common metabolic disease with a rising global prevalence, is associated with long-term complications [1]. The disease is one of the most important public health challenges for the $21^{\text {st }}$ century. More than 150 million adult persons are affected worldwide, and the number is expected to double in the next 25 years. The increase will be much higher in developing countries. For instance, between 1995 and 2025 , the number of diabetic patients is expected to increase by $170 \%$ in the developing countries, compared to only $42 \%$ in developed ones [2].

Diabetes mellitus is a group of metabolic diseases characterized by hyperglycemia that result from defects in insulin secretion, or action, or both. The prevalence of type 2 diabetes mellitus is rapidly increasing all over the world. The number of people with diabetes is increasing due to population growth, aging, urbanization, and increasing prevalence of obesity and physical inactivity [3].

Insulin Resistance occurs as a result of disturbances in lipid metabolism and increased levels of circulating fatty acids that accumulate within the insulin sensitive tissues such as muscle, liver and adipose tissues. Increased fatty acid flux has been suggested to be strongly associated with insulin resistant states such as obesity and type 2-diabetes [4]. Insulin is one of the most extensively studied proteins in many fields. Efforts in studying the molecule have been recognized widely, beginning with the Nobel Prize in 1923 to Banting and Macleod for the discovery of insulin [5].

\section{Types of Diabetes}

Type 1 diabetes when a person has diabetes, either: their pancreas does not produce the insulin they need. A perfect Glycemic control in T1D is achieved in less than one-third of children only, the major challenge being a steady supply of basal insulin that mimics the physiological endogenous pancreatic insulin secretion [6]. Most cases of Type 1 diabetes mellitus (T1DM) are thought to result from selective autoimmune destruction of pancreatic beta cells. In keeping with the autoimmune etiology, other autoimmune diseases occur with increased frequency [7].

Type-2 diabetes their body cannot use this insulin effectively - is the most common form of the disease typically afflicting obese adults. Patients with type-2 diabetes may be able to control their disease through diet and exercise alone or may require oral medications and/ or insulin injections. The recommendations for initial treatment of type 2 diabetes include lifestyle adjustments and oral anti-diabetic medications [8].

Type 2 Diabetes is a progressive disease and deterioration of Glycemic control is partly due to a gradual loss of $\beta$-cell function and mass .Currently, pharmacological agents for treating type 2 Diabetes comprises drugs acting on insulin-resistant organs or on the pancreas [9].

\section{Glycemic control}

Glycosylated hemoglobin (HbAlc) as a marker for average blood glucose levels over the previous months prior to the measurement is an important predictor of diabetes complications The role of improved Glycemic control in reduction micro vascular and neurological complications of diabetes was demonstrated in many observational and randomized controlled clinical trials [10].

\section{Hypoglycemia}

Recently, weekly blood glucose and, more importantly, Glycemic variability have been explored as means of assessing Glycemic control and altering management in type 2 diabetes [11]. Low blood glucose or hypoglycemia is the most common immediate health problem for patients with diabetes. Therefore, there is an urgent need to innovate new approaches that will sustainably stabilize the metabolic syndrome, or to an even greater extent, restore metabolic health; preferably, without requiring severe lifestyle curtailment and escalating doses of pharma therapeutic agents [12].

It occurs when the body gets too much insulin, too little food, a delayed meal, or more than the usual amount of exercise.

Corresponding author: Srilatha B, Presidency College, Bangalore University, India; E-mail: Srilatha.biotech09@gmail.com

Received August 08, 2011; Accepted September 11, 2011; Published September 17, 2011

Citation: Srilatha B (2011) High Risk Factors of Cardiovascular Diseases in Type 2 Diabetes. J Diabetes Metab 2:164. doi:10.4172/2155-6156.1000164

Copyright: (c) 2011 Srilatha B. This is an open-access article distributed unde the terms of the Creative Commons Attribution License, which permits unrestricted use, distribution, and reproduction in any medium, provided the original author and source are credited. 
Recognizing the determinants of poor Glycemic may contribute to a clearer understanding of modifiable antecedents of diabetes-related complications and may help to achieve improved Glycemic control and improve patient function and outcomes [3].

\section{Hyperglycemia}

Hyperglycemia occurs when the body gets too little insulin, too much food, or too little exercise; Diabetes mellitus (DM) refers to a group of common metabolic disorders that share the phenotype of hyperglycemia. Depending on the etiology of the DM, factors contributing to hyperglycemia include reduced insulin secretion, decreased glucose utilization, and increased glucose production [13].

Hyperglycemia is associated with excessive free radical generation and oxidant stress. Oxidant stress may be linked to diabetes via altered $\mathrm{NO}$ production and action. NO, an endothelium-derived relaxing factor is a pleiotropic intercellular messenger regulating many cellular functions [14]. The diabetes complications are equally associated with the both types of DM. However, the severity of the disease is related to the long term exposure to uncontrolled Glycemic [15].

Diabetic patients have reduced $\mathrm{Na}^{+} / \mathrm{K}^{+}$ATP as activity resulting in elevated intracellular $\mathrm{Ca}^{2+}$ concentration and platelet hyperactivity. Hyperglycemia is associated with increased PKC activity [16], and superoxide anion production as well as reduced antioxidant (glutathione) levels and NOS activity.

\section{Long Term Effects of Diabetes}

Diabetes is a debilitating disorder world-wide and a root cause for several serious complications such as cardiovascular diseases, cerebro vascular diseases, renal disorders, inflammation and immunity, and obesity. Insulin, the protein hormone synthesized by the $\beta$-cells of the pancreas is one of the prime hormones is responsible for the glucose uptake/utilization by the cells in the body and also tightly maintains the homeostasis of the glucose in circulation [17]. T2DM represents about $90-95 \%$ of all the diabetic populations and can be unchained by several factors such as the obesity, high calorie diet and lack of physical activity [18]. Numerous studies have demonstrated that statins reduce the incidence of stroke in patients at high risk for cardiovascular events [19].

Micro vascular complications include effects on small vessels, including arterioles, capillaries and venues. The development of these complications starts early and accounts for morbidity in the form of retinopathy, neuropathy and nephropathy in Type $2 \mathrm{DM}$ The present study was to determine prevalence and focused on presence risk factor affecting on diabetic vascular complications among type 2 diabetic outpatients in tertiary center [20].

Attempts to improve Glycemic control reduce the incidence of macro vascular events both in type 1 and type $2 \mathrm{DM}$ [21].The incidence and progression of micro vascular complications correlate with the Glycemic control and lower glycated hemoglobin (HbA1c) levels are associated with the reduction of cardiovascular risks [22].

\section{Diabetes and Specific CVD}

While death rates of cardiovascular disease have been in decline for the past 30 years, it remains one of the highest causes of mortality in the United States, accounting for 1 in every 2.9 deaths annually. Each day, 2400 Americans will die of cardiovascular disease, which amounts to 1 death every 37 seconds [23].

Cardiovascular disease is the leading cause of morbidity and mortality in patients with diabetes mellitus. Patients with diabetes mellitus have a 2 to 4 time's higher risk of cardiovascular disease and up to a 3 times increase in mortality than non diabetics. The accelerated rate of atherosclerosis seen in diabetes mellitus predisposes patients to coronary artery disease and to higher rates of myocardial infarction and death [24]. Common risk factors were documented including high blood pressure, diabetes, previous stroke and myocardial infarction, cardiovascular disease, hyperlipidaemia, atria fibrillation, periphery artery occlusive disease (PAOD), current smoking and alcohol consumption [25].

\section{Myocardial infarction}

Myocardial infarction is a rare complication of valve surgery, most commonly mitral valve replacement [26].Myocardial infarction rates are increased among diabetics of all ages. The prevalence of unrecognized myocardial infarction and silent myocardial ischemia detected by a treadmill exercises stress test was increased in patients with diabetes mellitus [27].

Myocardial viability represents a state of impaired contractility with the potential for recovery when blood supply is adequately restored. Traditionally, the evaluation of regional myocardial function was performed by visual assessment of the degree of thickening and inward displacement of individual myocardial segments. Obviously limited by inter and intra observer variability, poor visualization of some myocardial segments [28]. Three acute large vessel stroke patients presents to the emergency department [29].

\section{Atherosclerosis}

Cardiovascular diseases (CVD) remain the leading cause of death in the US despite the efforts in prevention and treatment. Low plasma HDL cholesterol (HDL-C) is consistently associated with increased risk of atherosclerotic disease. Assessment of apolipoproteins, lipoprotein particle size and number allows a further characterization of CVD risk [30].

Obesity is associated with an increase risk for cardio metabolic diseases such as atherosclerosis and type 2 diabetes [31]. Atherosclerosis is characterized by chronic inflammation affecting the arterial intima. Therefore certain cytokines and chemokines can be considered markers of CVD risk as well.

\section{Lipoproteins}

High-density lipoproteins, which transport cholesterol away from arteries, are protective. Low-density and very low-density lipoproteins, in contrast, can penetrate the arterial wall and deposit cholesterol within the artery, contributing to heart disease [32].

Atrioventricular (AV) block can be defined as a delay or interruption in the transmission of an impulse from the atria to the ventricles, and can be transient or permanent. It has been reported many causes for AV block including drugs, electrolyte disturbances, infiltrative malignancies, hyperthyroidism, and rheumatologic disorders.

Congestive heart failure and myocardial infarction are frequently reported cardiac signs and symptoms due to the accumulation of glycosphingolipids in vascular endothelial and smooth muscle cells and in cardiomyocites [33].

Atherosclerosis does not increase the systemic vascular resistance, but can generate localized plaques that can trigger sudden occlusions of coronary arteries by thrombus formation, unlike the gradual narrowing of the lumen of the pulmonary vasculature [34]. 


\section{Osteoporosis}

The number of patients with diabetes mellitus and osteoporosis is rapidly increasing in industrialized countries where Western-style aging societies are prevalent. Although the incidence of osteoporosis and type 2 diabetes mellitus is known to increase in prevalence with aging, both diseases were traditionally viewed as separate entities. However, the relationship between diabetes and osteoporotic fractures is recently and increasingly becoming recognized [35].

Normal serum Zinc and good Zinc dietary intake alone is not enough to improve osteoblastic function, but optimization of glucose metabolic profile in addition may help to improve osteoblasts activity and prevent bone complications [36].

\section{Cardiovascular Risk Factors in Type 2 Diabetes}

Individuals with type 2 diabetes are at higher risk of cardiovascular diseases (CVD) than those without type 2 diabetes. Diabetes is a chronic disease with severe complications and is a growing health issue in Europe and Sweden as well as in other parts of the world. The prevalence of diabetes in Sweden, mainly type 2, is about $4.5 \%$. For Diabetes hypertension and obesity is well-known major and independent cardiovascular risk factors [37].

For insulin sensitization, metformin is a commonly used drug for treating type 2 diabetes. As against these sensitization drugs, diet plays an important role in controlling glucose levels. Fiber foods and gums were found to bring glycemic control in diabetic subjects [38].

\section{Unhealthy diet}

Type 2 diabetes mellitus is a chronic disease with severe late complications and high mortality. The increasing prevalence of Type 2 diabetes is mainly due to reduced physical activity and consumption of unhealthy food and larger portion sizes in genetic susceptible individuals [39] and present in every country regardless of their stage of development, and afflicts people in all socio-economic classes.

Long term complications of diabetes include effects on the eyes, kidneys nerves, and blood vessels that can lead to blindness, neuropathy and Hypertension with organ damage is frequently observed [40].

\section{Heavy alcohol consumption}

Heavy alcohol intake increases the risk of hypertension and cardiovascular disease, but the relationship between alcohol consumption and type 2 diabetes remains controversial [41]. Some studies suggested no association between alcohol intakes and type 2 diabetes. Systolic and diastolic heart function, myocardial creatine phosphokinase leakage and myocardial antioxidants after ischemia/ reperfusion were examined [42].

Other studies have shown that heavy drinking can increase blood glucose level or risk of type 2 diabetes .Some epidemiologic studies [43] have showed that proper quantities of alcohol consumption was a protective factor for coronary heart disease.

Mechanisms of protective effect of proper quantities of alcohol consumption on type 2 diabetes may be the same as on coronary heart disease. This protective effect on type 2 Diabetes or coronary heart disease may be explained by a viewpoint that moderate alcohol consumption can increase insulin sensitivity and high HDL-C.

\section{Dyslipidemia}

Type of dyslipidemia that is most characteristic of type 2 diabetic subjects is elevated triglycerides and decreased HDL cholesterol levels, although all lipoproteins have compositional abnormalities. Therefore, the primary target of therapy in diabetic patients is lowering LDL cholesterol (or possibly, non-HDL cholesterol). The goal here is weight reduction and increased exercise. However, for selected patients, combining a fibric acid (or low-dose nicotinic acid) with a statin also can be considered [44].

\section{Obesity}

Obesity is the most important modifiable risk factor for type 2 diabetes mellitus and most patients with diabetes are overweight or obese. It is well known that excess bodyweight induces or aggravates insulin resistance, which is a characteristic feature of type 2 diabetes [45]. Over $30 \%$ of surgical patients are obese (BMI>30) and it is estimated that over ten million surgeries involving obese patients will be performed in 2010 (National Hospital Discharge Survey data) [46].

Obesity is associated with the development of a broad group of malignant, metabolic, cardiovascular, and neurological disorders. Of importance in the treatment of and prevention of diabetes mellitus, myocardial infarction, peripheral vascular disease, and cerebral infarction, obesity is a major factor in the development of metabolic syndrome. However, potential explanations for this rise in the prevalence of obesity include a sedentary life style, increased number of daily meals, the presence of an eating disorder, a changing job market, or an effect of a yet unidentified environmental agent or exposure [47]

To determine the proportion of patients with Type 2 diabetes mellitus (T2DM) who lost 5-10\% of their body weight as part of their initial management. To determine associations between metformin uses, it is advised for dietary and physical modification on weight loss [48].

\section{Hypertension}

Patients with comorbid hypertension and type 2 Diabetes are common, have a greatly increased risk of premature cardiovascular and renal morbidity and mortality, and are likely to increase substantially in number over the next 10-15 years. We suggest the need for more aggressive management strategies for these patients, regardless of their baseline blood pressure, including the early use of combination therapy with blockers of the renin-angiotensin system [49]. Hypertension was seen in $32 \%$ of diabetic patients in this study which was similar to the previous study of $32.1 \%$ at the end of 2007 in the same center [50]. High blood pressure (HBP) is the leading cause of end-stage renal disease. Hypertension and obesity have been regarded as the most common cause of increased mass in general population [51].

Aggressive blood pressure control may be the most important factor in preventing adverse outcomes in patients with type 2 diabetes. The Appropriate Blood Pressure Control in Diabetes (ABCD) Trial is a prospective randomized blinded clinical trial that compares the effects of intensive versus moderate blood pressure control on the incidence and progression of type 2 diabetic complications.

\section{Lifestyle}

Patients with type 2 diabetes often have negative self-concepts, feel hopeless and, therefore, become lax about following their regimen. This study shows that quality of life education can have positive effect on diabetes self concept, and prevent physical and side effects of type 2 diabetes [52].

Affective and behavioral neuropsychiatric disturbances are 
frequently encountered in post-stroke patients [53]. Type 2 diabetes affects approximately 8 percent of adults in the United States. Some risk factors--elevated plasma glucose concentrations in the fasting state and after an oral glucose load, overweight, and a sedentary lifestyle--are potentially reversible. All diabetic patients if given proper education and guidance regarding foot care would be able to make a significant improvement in their life style which is helpful to avoid amputations [54].

\section{Conclusion}

It is well known that type 2 diabetes is frequently associated with cardiovascular risk factors (CVRF), such as hypertension and dyslipidemia, and that the prevalence of other factors, such as smoking, sedentary lifestyle, and obesity is greater than in the non diabetic population. The objective of this study was to describe the association of type 2 diabetics with modifiable cardiovascular risk factors and the degree of control of these in a population of type 2 diabetics. Integrated and multifactorial intervention in type 2 diabetic patients can achieve clinically significant reductions in cardiovascular diseases.

\section{References}

1. Chen D, Huang H, Xing Y, Liu Y, Xu Y, et al. (2011) A New Vanadium Complex Improves the Spatial Learning and Memory by Activation of Caveolin- MAPKCREB Pathway in Diabetic Mice. J Diabetes Metab 2:114

2. Ali ZH (2011) Health and Knowledge Progress among Diabetic Patients after Implementation of a Nursing Care Program Based on Their Profile. J Diabetes Metab 2:121

3. Al-Akour NA, Khader YS, Alaoui AM (2011) Glycemic Control and Its Determinants among Patients with type 2 Diabetes Mellitus Attending a Teaching Hospital. J Diabetes Metab 2:129.

4. Ragheb R, Medhat IS (2011) Mechanisms of Fatty Acid-Induced Insulin Resistance in Muscle and Liver. J Diabetes Metab 2:127.

5. Ramachandra S (2011) Do we need yet Insulin? J Diabetes Metab 2:0e4

6. Saini A, Devidayal, Verma S, Bhalla AK (2011) Comparative Efficacy of Once Daily Insulin Glargine with Twice Daily NPH Insulin in Children with Type 1 Diabetes. J Diabetes Metab 2:124.

7. Joffe B, Distiller L, Landau S, Blacking L, Klisiewicz A (2010) Spectrum of Autoimmune Disorders in Type 1 Diabetes - A Cross-Sectional Clinical Audit. J Diabetes Metab 1:112

8. Parvaresh E, Esteghamati A, Nakhjavani M, Aminorroaya A, Aboutorabi R, et al. (2011) Biphasic Insulin Aspart 30 (BIAsp 30) is Safe and Improves Glycemic Control in Insulin Naïve Patients with Type 2 Diabetes. J Diabetes Metab 2:123.

9. Fouqueray $\mathrm{P}$, Leverve X, Fontaine E, Baquié M, Wollemi C, et al. (2011) Imeglimin - A New Oral Anti-Diabetic that Targets the Three Key Defects of type 2Diabetes. J Diabetes Metab 2:126.

10. Al-Akour NA, Khaddar YS, Alaoui AM (2011) Glycemic Control and Its Determinants among Patients with type 2 Diabetes Mellitus Attending a Teaching Hospital. J Diabetes Metab 2:129.

11. Lemos Costa TMR, Detsch JM, Pimazoni-Netto A, de Almeida ACR, SztalMazer S, et al. (2011) Glycemic Variability and Mean Weekly Glucose in the Evaluation and Treatment of Blood Glucose in Gestational Diabetes Mellitus; Evidence for Lower Neonatal Complications. J Diabetes Metab 2:137.

12. Ong YC, Su LH, Zaini A (2011) Reversal of Metabolic Dysfunction through Polyvalent Pharmacotherapy-augmented Lifestyle Intervention: Case Reports. J Diabetes Metab 2:133.

13. Shanker JH, Mahmood SE, Joshi MC, Shaifali I (2011) Obesity Indices amongs Diabetics in an Urban Population of Western Nepal. J Diabetes Metab 2:134.

14. El Asrar MA, Adly AAM, El Hadidi E, Gharib M (2011) Serum and Urinary Nitrites and Nitrates and Doppler Sonography in Detection of Early Diabetic Complications. J Diabetes Metab 2:117.

15. da Silva SB, Costa JP, Pintado ME, Ferreira DC, Sarmento B (2010) Antioxidants in the Prevention and Treatment of Diabetic Retinopathy - A Review. J Diabetes Metab 1:111.
16. Emara E, Abdel-Sater KA (2011) Beneficial Effects of Calcium Channel Blocke "Nifedipine" on Abnormalities of Platelets and Lipid Metabolism in Patients with Type II Diabetes Mellitus. J Diabetes Metab 2:131

17. Uppu RM, Parinandi NL (2011) Insulin Sensitization and Resistance Interrelationship Revisited with a Quantitative Molecular Model Approach. J Diabetes Metab 2:106e.

18. Ribeiro C, de Alencar Mota CS, Voltarelli FA, de Araújo MB, Botezelli JD, et al. (2010) Effects of Moderate Intensity Physical Training in Neonatal AlloxanAdministered Rats. J Diabetes Metab 1:107.

19. Lai HM, Aronow WS, Mercando AD, Kalen P, Desai HV, et al. (2011) A Case of 2:1 Atrio -Ventricular Block in Digoxin Toxicity. J Clinic Experiment Cardiol $2: 156$

20. Abougalambou SSI, Hassall MA, Suleiman SAS, AbougalambouAS (2011) Prevalence of Vascular Complications among Type 2 Diabetes Mellitus Outpatients at Teaching Hospital in Malaysia. J Diabetes Metab 2:115.

21. Stettler C, Allemann S,JüniP, Cull CA, Holman RR (2006) Glycemic control and macrovascular disease in types 1 and 2 diabetes mellitus: Meta-analysis of randomized trials 152: 27-38.

22. Esteghamati a, Nakhjavani M, Aminorroaya a, Aboutorabi R, M Niafar, etal. (2011) Biphasic Insulin Aspart 30 (BIAsp 30) is Safe and Improves Glycemic Control in Insulin Naïve Patients with Type 2 Diabetes. J Diabetes Metab 2:123.

23. Duran JM, George JC (2011) A Review of the Basis of Autologous Stem Cell Therapy for Coronary Artery Disease. J Clinic Experiment Cardiol 2:130

24. Li YW, Aronow WS (2011) Diabetes Mellitus and Cardiovascular Disease. J Clinic Experiment Cardiol. . 2:114

25. Broussalis E, Kunz AB, Luthringshausen G, Ladurner G, Trinka E, et al. (2011) Gender Differences in Patients with Intravenous Thrombolytic and Conservative Treatment for Acute Ischemic Stroke. J Neurol Neurophysiol 2:117.

26. Vaquerizo B, Serra A, García-Picart J (2011) Perioperative ST-segment Elevation Myocardial Infarction during Mitral Valve Annuloplasty: Role of Early Angiography. J Clinic Experiment Cardiol 2:136

27. Li YW, Aronow WS (2011) Diabetes Mellitus and Cardiovascular Disease. $J$ Clinic Experiment Cardiol. . 2:114

28. M, Kashlan H, et al. (2011) Usefulness of Resting Strain Rate Imaging to Predict Viability following Acute Myocardial Infarction Strain Rate Imaging and Myocardial Viability. Translational Medic 1:101.

29. Dababneh H, Guerrero W, Wilson K, Hoh BL, Mocco JD, et al. (2011) Observation of Mean Transit Time (Mtt) Perfusion Maps on a 320-Detector Row Ct Scanner and its Potential Application in Acute Ischemic Stroke. J Neurol Neurophysiol 2:117.

30. Calle MC, Vega-Lopez S, Segura-Perez S, Volek JS, Pérez-EscamillaR, et al. (2010) Low Plasma HDL Cholesterol and Elevated C Reactive Protein furthe Increase Cardiovascular Disease Risk in Latinos with Type 2 Diabetes. J Diabetes Metab 1:109.

31. Lavoie M, Rabasa-Lhoret R, Ziai S, Lavoie J (2011) Blood Glutathione Peroxidase Activity in Relation with the Risk of Cardiovascular Diseases in Obese Women. J Diabetes Metab 2:136.

32. Mikirova NCascara J, Hunninghake R, Riordan N (2011) Increased Level of Circulating Endothelial Micro particles and Cardiovascular Risk Factors. J Clinic Experiment Cardiol 2:131.

33. Serra W, Fagnani S, Ardissino D, Gherli T (2010) Late-Onset Cardiac Variant of Fabry Disease Responsive to Short-Term Treatment with Agalsidase Alpha. $\mathrm{J}$ Clinic Experiment Cardiol 1:109.

34. Guntheroth WG (2010) Increased Pulse Pressure Causes Vascular Injuryin Pulmonary and Systemic Arteries. Decreasing the Pulsatility with Banding andVasodilators Can Stabilize Pulmonary Hypertension. J Clinic Experiment Cardiol1:107.

35. Kanazawa I (2011) Osteocalcin Possesses Hormonal Function Linking Bone to Glucose Metabolism. J Diabetes Metab 2:105e.

36. Abo-El-Asrar M, Farid SM, Maraghy MOE, Mohamedeen AK (2011) Serum Osteocalcin, Zinc Nutritive Status and Bone Turnover in Children and Adolescents with Type1 Diabetes Mellitus. J Diabetes Metab 2:128.

37. Taloyan M, Saleh-Stattin N, Johansson SE, Agréus L, Wand ell P (2010) 
Differences in Cardiovascular Risk Factors in Swedes and Assyrians/Syrians with Type 2 Diabetes: Association with Lifestyle-Related Factors. J Diabetes Metab1:110.

38. Ramulu P, Giridharan NV, Udayasekhararao P, Janardanasarma MK (2011) Insulin Sensitization and Resistance Interrelationship in a Prediabetic Rat: A Quantitative Molecular Model. J Diabetes Metab 2:140.

39. Vadstrup E, Frolich A, Periled H, Borg E, Roder M (2009) Lifestyle intervention for type 2 diabetes patients - trial protocol of The Copenhagen Type 2 Diabetes Rehabilitation Project. BMC Public Health 9: 166

40. Seck SM, Cisse MM, Ka EF, Diallo I, Gueye S, et al. (2011) Hypertension in Black Africans with Autosomal Polycystic Kidney Disease. J Nephrol Therapeutic 1:102

41. Li H, Wang G, Wang A, Tong W, Zhang Y (2011) Alcohol Consumption and Risk of Type 2 Diabetes in Mongolian Population, Inner Mongolia, China. J Diabet Metabol 2:116.

42. Shen WL, Chen LB, Zhao JX, Guo SJ, Chen YC (2011) Effects of Hyperosmotic Sodium Chloride Perfusion on Ischemia/Reperfusion Injury in Isolated Hearts of Normal and Stroke-Prone Spontaneously Hypertensive Rats. J Clinic Experiment Cardiol 2:146.

43. Mukamal KJ, Chiuve SE, Rim EB (2006) Alcohol consumption and risk for coronary heart disease in men with healthy lifestyles. Arch Intern Med 166:2145-2150.

44. Steven M H (1998) MD Management of Dyslipidemia in Adults with Diabetes. Diabetes Care January 21: 160-178
45. Hauner $\mathrm{H}$ (2004) Managing type 2 diabetes mellitus in patients with obesity Treat Endocrinal 3: 223-232.

46. Gayes JM (2011) Obesity, Obstructive Sleep Apnea and the "HELP" Position. J Anesthe Clinic Res 2:115.

47. Majithia R, Koch TR (2011) Our Obesity Crisis Requires The Development of New, Widely Available Options: Can Yoga Function In A Major Role? J Yoga Phys Therapy 1:e102.

48. Mungrue K, Roper LA, Chung T (2011) Assessment of Weight Loss in the Management of Patients with Type 2 Diabetes Mellitus in Primary Care in Trinidad. J Diabetes Metab 2:120.

49. Lars R, Bernard W, Luis MR (2008) The management of the type 2 diabetic patient with hypertension - too late and too little: Suggested improvements 17 250-259

50. Mansour AA, Wanoose HL, Odaa AH (2011) A Three Year Cohort Prospective Type 2 Diabetes Control Study in Basrah. J Diabetes Metab 2:119.

51. Anwar AM, Mustafa MM, Nosir YFM (2010) Left Ventricular Remodeling in Diabetic Patients with and without Hypertension. J Diabetes Metab 1:108.

52. Samadi N, Safavi M, Mahmoodi M (2011) Impact of Quality of Life Education on Self-Concept among Type 2 Diabetes Patients. J Diabetes Metab 2:132.

53. Perez DL, Catenaccio E, Epstein J (2011) Confusion, Hyperactive Delirium and Secondary Mania in Right Hemispheric Strokes: A Focused Review of Neuroanatomical Correlates. J Neurol Neurophysiol S1.

54. Patra SR, Jahnavi G (2011) An Improvement in Compliance for Foot Care in Persons with Type 2 Diabetes with a Teaching Session. J Diabetes Metab 2:130. 